\title{
EPIDEMIC MYALGIA AFFECTING THE TRAPEZIUS MUSCLE
}

\section{DONALD WILSON}

In 1934 Sylvest published a monograph in Danish entitled "Epidemic Myalgia-Bornholm Disease" in which he described a morbid condition of the intercostal and abdominal muscles, outbreaks of which occur in sufficiently large numbers in certain localities to reach epidemic proportions. The term "epidemic myalgia" was used, on the suggestion of the late Sir George Buchanan, in order to avoid a slur upon the pleasant island of Bornholm. In his comprehensive monograph Sylvest collected all the known descriptions of this disease that had appeared before his own publication, and since that date outbreaks have been recognized more readily and epidemics have been described in most parts of the world. Furthermore, several outbreaks have been observed and recorded in which the trapezius muscle has been principally involved, and which have had an appreciable morbidity rate.

\section{Clinical Description}

The morbid condition to be described differs from Bornholm disease in several important features. Only one muscle seems to be affected, there is usually absence of pyrexia, and the only complication which has been commonly described is persistent fibrositis. This syndrome may have a sudden or insidious onset ${ }_{3}$ and pain is the most prominent feature, which may be maximal from the start, or which may increase rapidly in severity, reaching its maximum within a few hours of onset. It is persistent and is increased by any movement in which the affected muscle is required to contract. The pain may be lancinating or aching, radiating to occiput or down the arm; paraesthesia of the arm on the affected side has been recorded. Headache is usually occipital, and is common in both the prodromal and the fully developed stages. Pyrexia is unusual and, if present, never exceeds $100^{\circ} \mathrm{F}$. Catarrhal conditions of the upper respiratory tract have had a high incidence in some outbreaks. The condition may quickly resolve, but it more usually extends over a period of from eight to fourteen days, gradually diminishing in severity. During the course of the disease complications are exceptional, but chronic fibrositis may occur as a sequel.

\section{Description of Outbreaks}

In 1935 Massell and Solomon gave the results of a questionnaire issued to 300 people employed in hospitals.
Fifty-two gave answers admitting to pain in the region of the trapezius muscle during the previous two months; they attributed the pain either to exposure to a draught or to an abnormal position in the course of their work. The authors thought that this figure was significant enough to warrant the assumption that a common infective agent had been present. In addition they were able to prove that no abnormal position had been used in many cases where this had been claimed as a cause, and that the theory of draught could not be upheld as the sole aetiological factor.

In September, 1942, Beeson and Scott published an interesting paper on the results of a survey of 1,031 people who were drawn from an A.T.S. unit, a factory, and a hospital. Each unit had two surveys, and 125 cases were collected. These authors showed that there was an alteration in the incidence of the disease. Between September and November, 1941, the living-in personnel of the hospital had an attack rate of $23.6 \%$, and the living-out $11.6 \%$. In the second survey (March to April, 1942) these figures were reversed, the living-in personnel having an attack rate of $11.6 \%$ and the livingout $27.3 \%$. In their paper Beeson and Scott note that the trapezius was almost invariably involved, though other muscles might be tender. Pyrexia was not marked, though $41 \%$ had symptoms of a "common cold" at the onset. Tender nodes were present in the affected muscles in all cases. The erythrocyte sedimentation rate was not raised, and the white cell count usually showed a lymphocytosis. The authors described attempts to convey the disease by experimental methods; no definite results were achieved but it is significant that the incidence of myalgia was found to be higher in volunteers who received whole blood inoculation than in the remainder of the community.

Four other outbreaks have been observed in Service Units. Copeman (1944) has given particulars of two outbreaks which he observed early in 1942 . In the first the men were housed in a hutted camp and the outbreak occurred in January, 1942; eight men were affected in a company. Two men reported sick with stiff necks; ten days later two more reported sick with similar symptoms. When the camp was inspected four more men were found who were recovering from the same condition although they had not reported sick. The symptoms were pain in one side of the neck radiating down the arm. Recovery was slow but eventually complete. Only one had catarrhal symptoms at the onset. These men fed together in an adjacent hut, but only two slept in the same hut.

The second outbreak described by Copeman occurred in a hutted hospital during February, 1942. Eight members of the staff suffered from the complaint. The incubation period varied from eight to twenty-one days. The principal symptom was pain in the neck radiating 
down the arm. Paraesthesiae occurred in some cases, and mild muscular pains were found elsewhere ; but attention was focused on the trapezius. The erythrocyte sedimentation rate was raised in all cases, but the white cell count was within normal limits. When one case from this group was seen a year later he was suffering from persistent fibrositis.

In July and August, 1940, the writer had the opportunity to observe a similar outbreak, though facilities were lacking for a complete investigation. Two men reported sick complaining of pain in the left scapular region radiating towards the neck. Extreme tenderness was present in the trapezius at the vertebral border of the scapula; tender nodes were also present along the superior border of the trapezius. Both men were apyrexial, but complained of mild malaise which had been present.for the previous two or three days. During the next fourteen days twelve other men from the same unit reported sick with similar symptoms. In all cases the trapezius was the only muscle involved, though pain was complained of either in the neck or upper part of the back. One of the first two men who reported sick stated that he had just returned from leave, where he had been in contact with a brother who had similar symptoms. In this outbreak the pain appeared to originate from an exquisitely tender focus and to radiate over the back; any movement, such as bending, sudden movement involving the dorsal spine, coughing, sneezing, and hiccoughing, was very painful. (It should be mentioned that the weather was warm and sunny.)

In September and October, 1943, I was able to make fuller observation of an outbreak in a unit numbering 159. Twenty-nine cases occurred, including both women and men. Pain in the trapezius was an invariable finding; pain sometimes radiated down the arm, but paraesthesiae were not marked. Headache was a prodromal symptom in seventeen cases; catarrh of the upper respiratory tract was noted in seventeen cases during the initial stage. The erythrocyte sedimentation rate varied from 2 to 14 . There was no leucocytosis, and no monocytosis. From this outbreak at least two cases of persistent fibrositis resulted.

\section{Anatomy}

It is necessary to refer briefly to the anatomy of the trapezius in relation to the symptomatology of this syndrome. The trapezius is a large, superficial muscle covering almost the whole of the dorsal region of the back. It originates from the superior line of the occiput, the cervical and dorsal spines, and the inter-spinous ligaments; from this extensive origin the muscular fibres converge towards the shoulder, to be inserted continuously, from before backwards, in the outer third of the posterior surface of the clavicle, the inner border of the acromion process, and the spine of the scapula. Thus it will be seen that the trapezius will be involved in any movement of the head, neck, dorsal spine, or scapula. A painful condition of the trapezius may cause the complaint of pain in the neck, shoulder, or back.

\section{Aetiology}

In discussing the aetiology of this syndrome it is important to remember that a number of people involved in the outbreaks reviewed above had not thought their symptoms severe enough to warrant reporting sick, and it is possible that the actual rate is higher than the figures quoted, particularly in towns.
- Beeson and Scott (1942) quote a difierent incidence for the disease in various graups of people. In the hospital unit, during the first survey the living-in personnel had an incidence of $26 \%$ and in the second survey one of $11.3 \%$, whilst amongst the living-out personnel the incidence rate was doubled in the second survey. In the A.T.S. unit, which had twenty-four cases during the first survey, the week from December 30 to January 5 had by far the highest incidence; in the second survey only seven cases were found and the attack rate had fallen from $13.6 \%$ to $4.3 \%$. On further investigation it was found that fifteen cases out of twentyfour came from one dormitory. These figures are suggestive of an infective agent. Attempts to transmit the disease to humans and animals are described by these authors, and although their results are inconclusive there is some evidence that this condition is infective.

In the first outbreak observed by Copeman two cases slept in the same hut but all fed in the same mess. In his second series, which occurred in a hutted hospital, eight members of the staff were affected.

Claims are made by patients and others that the sudden onset of stiff neck is due either to draughts or to an unusual posture. It is probable that in sporadic cases either of these may be the cause of the pain. Before the war of 1939-45 there was correspondence in a medical journal about "sunshine roof" shoulder, a condition in which patients complained of severe pain in the trapezii, sudden in onset, after a drive in a car with the sunshine roof open. In many light cars when the roof is opened a swift down-draught of air is directed on to the shoulders and neck of the occupiers of the front seats. It is known that a rapid rush of air has a marked cooling effect on the skin; if this is localized to one area, which may be insufficiently protected, there may be a spasm of the underlying superficial muscle, which in this case is the trapezius. It is generally recognized that use of a muscle in an unaccustomed posture may be a cause of pain in the muscle, probably owing to spasm; but the pain is usually aching in character and can be relieved by relaxation. This group, which includes the common type of shoulder pain seen amongst housewives, can be differentiated by a careful history of the pain and the activities of the complainant.

In 1942 Houghton and Jones described an outbreak of epidemic or infectious myalgia secondary to streptococcal sore throat; six members of a nursing staff had generalized muscle pains after initial pyrexia; headache was present in all cases and was very severe. The pain was not relieved by salicylates. The streptococcus recovered from the throats were of different types. The sedimentation rate was slightly raised and the white cell count showed an increase in monocytes. The findings in this outbreak are compatible with a virus infection. Houghton and Jones considered that this outbreak had similarities both to trench fever and to Bornholm disease, and they thought it might be due to a 
myotrophic virus. A similar epidemic of myositis was described by Williams in 1941. In his cases evidence of neuritis and meningeal symptoms were found; again the causative organism was assumed to be a virus.

On the assumption that there may be myotrophic types of virus capable of causing various degrees of illness, it is probable that myalgia of the trapezius may be a virus type of infection which is capable of causing epidemics. The extent of the outbreaks is difficult to determine, because many people who have contracted the condition do not report it, thinking it will pass away in a short time. Usually it causes disability for a few days and then recovery is complete, but in some a persistent fibrositis results, causing a great loss of efficiency in the affected person.

\section{Differential Diagnosis}

Bornholm Disease.-Pain, usually situated in the lower intercostal and upper abdominal muscles of one side, is the principal feature. This pain is very severe and tends to be paroxysmal, as noted by Pickles (1933) in his account of the disease as it occurred in a Yorkshire dale. In addition to the intercostal muscles, pain has been described as occurring in the lumbar, pectoral, and biceps muscles. Localized areas of tenderness can be felt in the affected muscles, and the spontaneous pain which is so characteristic corresponds to these tender areas.

Polymyositis.-Polymyositis has been described by Gowers (1899), Langmead (1923), and Somers (1939), as being slowly progressive, with lack of severe pain and, eventually, with paralysis of the affected muscles. The condition may also follow the exanthemata, enteric fever, and tuberculosis. Other writers have described an acute recurrent polymyositis; most of the cases showed a high degree of eosinophilia which was not due to trichiniasis.

Neuritis.-At the onset, cervico-occipital neuritis may resemble myalgia of the trapezius very closely; but usually the tender nodes in the muscle are absent and the pain is not limited to the region of the trapezius.

Fibrositis.-In considering the differential diagnosis of fibrositis involving the trapezius, neuritis of the cervical plexus and the causes of pain referred along the distribution of the plexus must be considered. In neuritis of the great occipital nerve, which may be caused according to Wilson (1940) by such excitants as chill, wetting, or currents of cold air playing on the part, there is tenderness along the course of the nerve combined with spontaneous pain which is increased by movement; the pain usually starts in the region of the occiput and radiates up to the vertex of the scalp. The pain due to fibrositis is not so widespread, and nerve tenderness is not so marked. Neuritis of the long thoracic nerve causes an aching pain at the lower angle of the scapula, and neuritis of the suprascapular causes pain above the spine of the scapula which is followed by wasting of the muscles supplied by the involved nerve.

\section{Treatment}

Treatment of this condition is palliative. In some cases radiant heat, infra-red rays, etc., alleviate the pain. Salicylates appear to have no effect. In very severe cases, supporting the arm by means of a sling in order to relieve the trapezius from the weight of the arm procures temporary alleviation. Injection of the tender nodes with procaine does not bring the degree of relief of pain usual in fibrositis.

\section{Conclusion}

An attempt has been made to describe myalgia of the trapezius which occurs in small epidemics and may be due to a virus. The characteristics of this condition are the sudden apyrexial onset of persistent pain, varying in severity and degree of disability; the condition may be followed by persistent fibrositis.

Bornholm disease is contrasted with myalgia of the trapezius.

This condition may be the cause of pain in the side of the neck and arm, and the recognition that it may be infective is important in preventing the spread of the disease through communities and the resulting loss of man-hours of work.

\section{REFERENCES}

Beeson, P., and McNair Scott, T. F. (1942). Proc. roy. Soc. Med., $35,733$.

Copeman, W. S. C. (1944). Personal Communication.

Gowers, W. R. (1899). Brit. med. J., 1, 65.

Houghton, L. E., and Jones, E. I. (1942). Lancet, 1, 196.

Langmead, F. S. (1923). Arch. Pediat., 40, 112.

Massell, B. F., and Solomon, P. (1935). New Engl. J. Med. 213, 399.

Pickles, W. N. (1933). Brit. med. J., 2, 817.

Somers, D. C. (1939). J. Bone Jt. Surg., 21, 414.
Sylvest, E. (1934). "Epidemic Myalgia." London.

Wylvest, E. (1934). "Epidemic Myalgia." London.

Wilson, S. A. K. (1940). "Neurology." 'London. 